\title{
Cochrane review questions value of industry-funded clinical trials
}

$\mathrm{I}$ $\mathrm{t}$ appears that efforts made in recent years to reduce bias in indusfruitless.

"The changes that have been made in the last decade haven't made any difference," says Dr. Joel Lexchin, a health policy professor at York University in Toronto, Ontario, and one of the authors of Industry sponsorship and research outcome, a new review from the Cochrane Collaboration (http://online library.wiley.com/doi/10.1002/14651858 .MR000033.pub2/pdf).

It is well known that the odds of clinical trials producing findings favourable to the drug industry are higher in research sponsored by industry rather than independent bodies. Recent efforts to curb funding bias include clinical trial registries, disclosure of raw data and an increased focus on transparency by medical journals regarding authors' financial ties to industry.

The Cochrane review, which considered 48 studies and expanded upon a previous paper to also include research on medical devices, explores if these initiatives have reduced bias. The answer appears to be a resounding "no," according to coauthor Sergio Sismondo, a philosophy professor at Queen's University in Kingston, Ontario.

"All the evidence I've seen says the bias is the same. It seems to be entirely consistent with what it was a decade ago," says Sismondo, who suggests there are few effective safeguards against industry bias except "extra-vigilant peer review," which is exhaustive work.

The persistence of bias in companysponsored clinical trials is a growing cause for concern, the authors note, because industry's influence is increasing. Since the 1980s, the bulk of medical research conducted in the United States has been paid for by private companies. The pharmaceutical industry spends more on research, primarily clin-

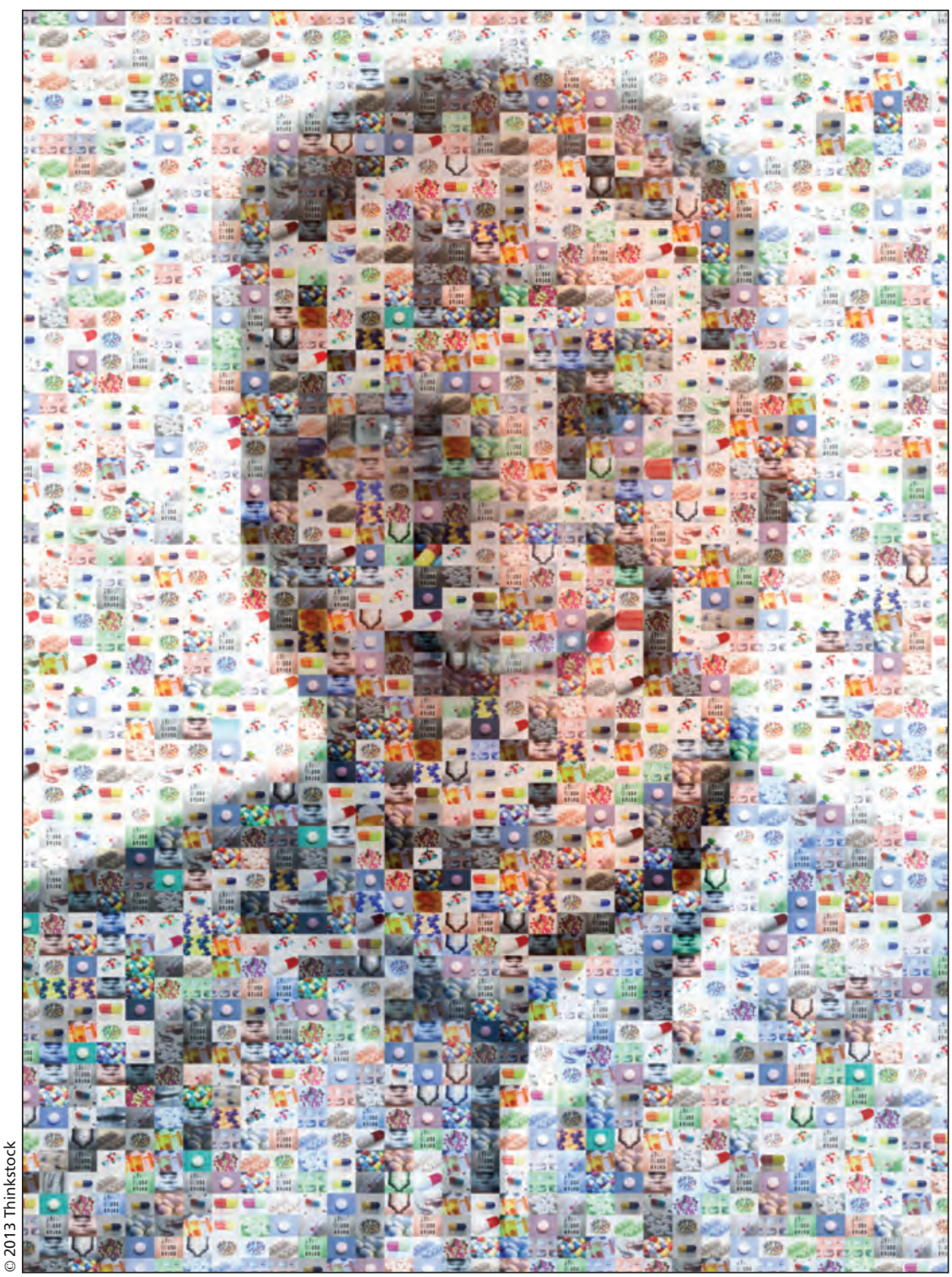

The persistence of bias in company-sponsored clinical trials is a growing cause for concern because industry's influence is increasing.

ical trials, than the National Institutes of Health. In Canada, approximately $80 \%$ of clinical trials are industry-funded, says Lexchin.

The findings from skewed research can have a direct impact on patients. "Clinical trials form the basis for how drugs are used in practice," says Lexchin. "In other words, doctors may choose drugs thinking that they're going to be more effective than they actually are."

Another problem that arises when biased trials come to light is that all industry-sponsored research comes under suspicion, even properly designed and well-run studies. Cautious physicians may end up dismissing legitimate 
research that could actually have improved their practices.

The review noted that bias doesn't always creep into clinical trials through the usual suspects. "What we found is that it's not based on the traditional things that are usually measured," says Lexchin. "So it's not due to the randomization process, its not due to double-blinding, it's not due to following dropouts or study size."

Companies may influence results in more subtle ways, by making small changes to how a trial is designed, reported or conducted. Perhaps the recruitment process favours participants likely to respond positively to a drug, for example, or a product is compared to an inappropriate competitor.

"If the trial is about preventing harm, the company could compare its product to one with known harms," says Sismondo.

To help reduce bias, all medical journal editors should have access to a trial's protocol, suggests Sismondo. A detailed plan of the tests and treatments in the study could help them determine whether the research is balanced. Drug and medical devices companies should also be compelled to pay for independent statistical analysis, he says.

Finally, governments and noncommercial sponsors should consider mak- ing independent trials a mandatory requirement for drugs and device approval by regulatory agencies, to shift away from trials with a marketing purpose.

"We've known for a long time [that] industry-funded studies tend to be biased. People should read studies funded by industries differently than they read independent studies," says Sismondo. "It does not mean we should throw away all industry-funded trials, but we should recognize there is probably going to be bias." - Veronique Hynes, Ottawa, Ont.

CMAJ 2013. DOI:10.1503/cmaj.109-4378 